\title{
Schwerpunkt: Finanzkrise
}

\section{Andreas Fisahn Europäische Union in der Legitimationskrise}

Die Welt befindet sich in einer Krise, welche die Feblkonstruktion der Europäischen Union offenbart. Diese ist angesichts der Krise handlungsunfäbig und muss sich auf Beschwörungsformeln beschränken, die Protektionismus und Renationalisierung abwebren. Gleichzeitig kümmern sich die Institutionen der EU und die Mitgliedstaaten wenig um die im Lissaboner Vertrag wieder beschworenen Regeln der Marktorthodoxie. Damit geraten zunächst die neoliberalen Spielregeln, möglicherweise aber auch die Institutionen der Europäischen Union selbst in eine Krise. Die Finanzmarktkrise hat sich zu einer Systemkrise ausgewachsen, welche die europäische Politik in absebbarer Zukunft bestimmen wird und die Krise der Europäischen Union zu einer Legitimationskrise werden lässt.

Legitimität wird nach einer bekannten Unterscheidung von Fritz Scharpf nicht nur über den Input, sondern auch über den Output des demokratischen Prozesses erzeugt. Die Input-Perspektive betont die Beteiligung der Bürger und die Verantwortlichkeit der Regierenden gegenüber den Regierten. Dagegen geht es beim Output um die sachliche Qualität der Politik. Beides zusammen schafft erst die nötige Integration des Gemeinwesens oder die Legitimität von Politik. ${ }^{1}$ Mit Blick auf beide Bedingungen der Legitimität befindet sich die Union gegenwärtig in einer schwierigen Situation.

\section{Die Krise und die Liberalisierung der Finanzmärkte im EU-Recht}

Mit der ökonomischen Krise rückt zunächst der Output europäischer Entscheidungsfindungen ins Blickfeld des Interesses. Die Finanzmarktkrise und die ihr folgende Rezession ist ein Produkt der marktradikalen Ordnung, die seit Maastricht in die europäischen Verträge eingeschrieben wurde und auch den Lissaboner Vertrag weiter bestimmt. In den Römischen Verträgen von 1957 sollten gemäß Art. 67 Abs. 1 EWG-Vertrag Beschränkungen des Kapitalverkehrs beseitigt werden, „soweit es für das Funktionieren des gemeinsamen Marktes notwendig ist". Die Liberalisierung des Kapitalverkehrs wurde danach also unter einen Erforderlichkeitsvorbehalt gestellt, was den Mitgliedstaaten gestattete, Kapitalverkehrsbeschränkungen beizubehalten. Dieses bedingte Postulat, den Kapitalverkehr zu liberalisieren, galt bis 1993.

Die geltende Regelung spricht eine deutlich andere Sprache. Im geltenden Art. 56 Abs. 1 EGV (Nizza Vertrag) heißt die Vorschrift gleichlautend mit Art. 63 Abs. 1 des AEUV als Teil des Lissaboner Vertrages: „Im Rahmen der

1 Scharpf, Demokratietheorie zwischen Utopie und Anpassung, 1970. 
Bestimmungen dieses Kapitels sind alle Beschränkungen des Kapitalverkehrs zwischen den Mitgliedstaaten sowie zwischen den Mitgliedstaaten und dritten Ländern verboten. (2) Im Rahmen der Bestimmungen dieses Kapitels sind alle Beschränkungen des Zahlungsverkehrs zwischen den Mitgliedstaaten sowie zwischen den Mitgliedstaaten und dritten Ländern verboten." Hier gibt es ein striktes, uneingeschränktes Verbot, das einhellig als Gebot zur Liberalisierung, als „umfassende Liberalisierungspflicht" ${ }^{2}$ des Kapitalverkehrs verstanden wird. Das bedeutet, es dürfen mit Blick auf den Kapital- und Zahlungsverkehr keine Verbote und keine Genehmigungsvorbehalte bestehen, solange diese nicht ausnahmsweise ${ }^{3}$ gerechtfertigt sind.

Die Kapitalverkehrsfreiheit gilt seit 1993 nicht nur innerhalb der EU, sondern die Verträge normieren diese auch für die Beziehungen der Mitgliedstaaten zu Drittstaaten. So heißt es mit Blick auf das gegenwärtige Krisenszenario in Art. 59 EGV (Art. 66 AEUV): „Falls Kapitalbewegungen nach oder aus dritten Ländern unter außergewöhnlichen Umständen das Funktionieren der Wirtschafts- und Währungsunion schwerwiegend stören oder zu stören drohen, kann der Rat mit qualifizierter Mehrheit [...] gegenüber dritten Ländern Schutzmaßnahmen mit einer Geltungsdauer von höchstens sechs Monaten treffen, wenn diese unbedingt erforderlich sind. " Das verdeutlicht die Bedeutung, die der Kapitalverkehrsfreiheit im Vertrag eingeräumt wird, weil diese auch gegenüber Drittstaaten nur ausnahmsweise - etwa zur Abwendung von Finanzkrisen - und dann nur befristet beschränkt werden kann. Gemäß Art. 57 Abs. 2 EGV (Art. 64 III AEUV) bedürfen Maßnahmen, „die im Rahmen des Gemeinschaftsrechts für die Liberalisierung des Kapitalverkehrs mit dritten Ländern einen Rückschritt darstellen“, der Einstimmigkeit. Dies stellt eine gegenwärtig fast nicht zu überwindende Hürde dar, die Kapitalverkehrsfreiheit gegenüber Drittstaaten zu beschränken.

Im Klartext heißt dies, dass die europäischen Verträge die Deregulierung der Finanzmärkte normiert haben, die die Dominanz der Finanzmärkte gegenüber der Realökonomie ${ }^{4}$ und damit das Entstehen von Spekulationsblasen ermöglicht haben. ${ }^{5}$ Während bis zu Beginn der 1990er Jahre unterschiedliche Formen von Finanzmarktregulationen einschließlich von Kapitalverkehrskontrollen existierten, wurden diese anschließend sukzessive abgebaut. ${ }^{6} 1994$ hat Griechenland als letzter Mitgliedstaat der Europäischen Union seine Kapitalverkehrskontrollen abgeschafft, seitdem gibt es in den Industrieländern der OECD keine Kapitalverkehrskontrollen mehr. ${ }^{7}$ Kapitalverkehrskontrollen werden etwas tautolo-

2 Groeben/Schwarze, Kommentar zu EUV und EGV, 6. Aufl. 2004, Art. 56 Rn. 30.

3 Explizite Abweichungsmöglichkeiten oder Ausnahmen sieht Art. 65 AEUV (58 EGV) vor. Danach dürfen die Mitgliedstaaten ihre Steuergesetze anwenden, diese dürfen sogar explizit diskriminierend sein. Abweichungen sind zulässig, um die Einhaltung von Rechtsvorschriften, insbesondere wiederum des Steuerrechts, zu sichern. Schließlich sind Abweichungen von der Verpflichtung zur Liberalisierung explizit zugelassen, wenn dies „aus Gründen der öffentlichen Ordnung oder Sicherheit gerechtfertigt“ ist. Daneben wird von der Literatur - ohne dass der EuGH dies für die Kapitalverkehrsfreiheit explizit formuliert hätte - angenommen, dass auch Beschränkungen im Interesse des Allgemeinwohls zulässig sind, soweit diese nicht diskriminierend wirken und verhältnismäßig sind. Allerdings müssten sehr wesentliche Interessen des Staates berührt sein (Borchardt, Die rechtlichen Grundlagen der Europäischen Union, 3. Aufl. 2006, Rn. 769; Groeben/Schwarze [FN. 2], Art. 56 Rn. 12 m.w.N.).

4 Selbstverständlich ist auch die Finanzwirtschaft keine virtuelle Angelegenheit und als Kreditwirtschaft eng mit Produktion und Dienstleistung verwoben. Die Unterscheidung bringt zum Ausdruck, dass sich die Finanzwirtschaft von der stofflichen Produktion und Reproduktion z.T. gelöst hat und selbstreferenziell Werte produziert, denen keine stofflichen Werte gegenüber stehen.

5 Altvater, Inflationäre Deflation oder die Dominanz der globalen Finanzmärkte, Prokla Nr. 134 (2004), 42 ff.; Stapelfeldt, Die Europäische Union - Integration und Desintegration, 1998, S. 140 f.

6 Fisahn/Niggemeyer, Bremsklotz EU-Recht, Zum absehbaren Scheitern der Finanzmarkt-Regulierung, Blätter für deutsche und internationale Politik, 2009, $88 \mathrm{ff}$.

7 Huffschmid, Politische Ökonomie der Finanzmärkte, 1999, S. 121. 
gisch definiert als administrative wie auch marktkonforme Maßnahmen zur Regulierung der Kapitalströme in und aus einem Land heraus. Dazu gehören Restriktionen von Krediten ins Ausland, des Handels mit Wertpapieren, des Devisenkaufs und -verkaufs, des Erwerbs von Unternehmensbeteiligungen und anderen handelbaren Rechten wie den sog. abgeleiteten Wertpapieren, Derivaten und Optionen.

Deutschland kannte nach dem Zweiten Weltkrieg unterschiedliche Formen der Finanzmarktregulation, die zu unterschiedlichen Zeiten oder nebeneinander existierten und z.T. abgeschafft und wieder eingeführt wurden. So gab es eine Genehmigungspflicht für die Verzinsung von Einlagen auf ausländischen Konten. Das Wertpapiergeschäft war in unterschiedlicher Intensität genehmigungspflichtig wie etwa der Erwerb inländischer Wertpapiere durch Gebietsfremde. Das war verbunden mit einer Kapitalertragssteuer (Kuponsteuer) für Gebietsfremde, die inländische Wertpapiere erwarben. Sog. Pensionsgeschäfte waren ebenso genehmigungspflichtig wie der Verkauf inländischer Wertpapiere an Gebietsfremde oder die Aufnahme von Krediten im Ausland und die Veränderung der „terms of payment“, verstanden als vorgezogener Einzahlungen und verzögerter Auszahlungen. Es gab zeitweise eine besondere Mindestreservepflicht ${ }^{8}$ auf Einlagen Gebietsfremder und eine Bardepotpflicht, um die Aufnahme von Auslandskrediten zu verteuern. ${ }^{9}$ Frankreich verfolgte im Devisengesetz von 1966 das Ziel einer Devisenbewirtschaftung, die mit einem umfangreichen Instrumentarium von Kapital- und Devisenkontrollen erreicht werden sollte. In Italien bestand eine Genehmigungspflicht von Devisen- und Kapitaltransfers ins Ausland, von der entsprechende explizite gesetzliche Ausnahmen erforderlich waren. ${ }^{10}$

Mit der Liberalisierung der Finanzmärkte auf der Grundlage der europäischen Verträge und internationaler Vereinbarungen wie den WTO-Regeln wurden die politischen Rahmenbedingungen geschaffen, die als Voraussetzung der gegenwärtigen Finanzmarktkrise gelten müssen. Wenn aber die „Liberalisierungspflichten“ des europäischen Primärrechts mit verantwortlich dafür sind, dass auf den Finanzmärkten wiederkehrend Spekulationsblasen entstehen, die tendenziell auch die Realwirtschaft gefährden, dann sind diese Normen nicht geeignet, den Krisenursachen, d.h. dem Spekulationshype, zu begegnen. Oder negativ ausgedrückt: Die Spielregeln des europäischen Rechts verhindern eine angemessene und effektive Krisenprävention.

\section{Re-Regulierung der Finanzmärkte: kein Projekt der EU}

Allerdings ist eine Re-Regulierung der Finanzmärkte politisch offenbar auch nicht gewollt. Es gibt einflussreiche pressure groups, die auch angesichts der Krise am System des finanzmarktgetriebenen Kapitalismus festhalten wollen, weil sie die Profiteure waren oder weil sie sich mit Haut und Haaren der Freihandelsideologie verschrieben haben. Als die US-amerikanische Finanzmarktkrise in Europa für alle spürbar wurde und die ersten Banken vor dem Konkurs standen, schwenkte selbst die CDU auf eine Regulationsrhetorik um, welche die gesellschaftliche Linke zunächst blass werden ließ. Am 20.9.2008 titelte das Handelsblatt: „Merkel fordert Regulation von Finanzspekulationen. - Bundeskanzlerin Merkel hat angesichts der derzeitigen Finanzmarktkrise eine internationale

8 Mindestreserven sind unverzinsliche Guthaben, die von den Geschäftsbanken bei der Zentralbank zu unterhalten sind und die in einem bestimmten prozentualen Verhältnis zu den Einlagen stehen.

9 Schmidt, Kapitalverkehrskontrollen und ihre Wirkung, 1977, S. 67 ff.

10 Weniger, Kapitalverkehrskontrollen im Europäischen Währungssystem, 1988, S. $110 \mathrm{ff}$. 
Regulierung der Finanzmärkte gefordert und die USA und Großbritannien kritisiert." Röttgen, parlamentarischer Geschäftsführer der CDU, gefiel sich ebenfalls in der Rolle des Regulators und verstieg sich in „hart aber fair“ zu der These, mit der Finanzmarktkrise sei die Stunde der Politik gekommen - nun könne das Primat der Politik gegenüber der Ökonomie wieder durchgesetzt werden. Es schien nicht unmöglich, zu einer Politik der regulierten Finanzmärkte zurückzukehren, selbst ein neues Bretton Woods ${ }^{11}$ wurde in Erwägung gezogen.

Inzwischen zeichnet sich ab, dass auf eine Re-Regulierung der Finanzmärkte weitgehend verzichtet und am Konzept des Freihandels festgehalten werden soll. Unter der Führung der Bundesrepublik scheinen sich die großen Mitgliedstaaten der Union darauf verständigt zu haben, beim Prinzip liberalisierter Finanzmärkte zu bleiben. Die Verantwortung wird auf die internationale Ebene verschoben, auf die G 20, die UNO oder den IWF. Die Regulierung der Finanzmärkte wird auf Transparenzregeln reduziert, die dann international beaufsichtigt und kontrolliert werden sollen.

Merkel forderte im Januar 2009 auf dem Weltwirtschaftsforum in Davos ebenso wie auf dem „Kolloquium Neue Welt, neuer Kapitalismus“ in Paris, dass die Kontrolle über die Finanzmärkte von einer Weltwirtschaftsregierung der UNO übernommen werden solle: „Jetzt können wir darüber diskutieren, ob wir bestehende Institutionen wie zum Beispiel den Internationalen Währungsfonds aufwerten und mit neuen Kompetenzen ausstatten wollen. [...] Oder aber wir entscheiden uns - auch das halte ich für eine Möglichkeit -, neben einem UNSicherheitsrat einen Wirtschaftsrat bei den Vereinten Nationen anzusiedeln, der etwas anderes tut als heute der ECOSOC, der sich tage-, monate- und jahrelang damit beschäftigt, nette Gutachten zu schreiben, aber letztlich keine prägende Kraft für die Ordnung der Weltwirtschaft ist. "12 Der Vorteil einer solchen Position ist, dass man „zu Hause“ weiter machen kann wie bisher und gleichzeitig einen UN-Wirtschaftsrat fordern und eine europäische Wirtschaftsregierung, wie sie Sarkozy vorgeschlagen hat, ablehnen kann. Die verbal eingeforderte ReRegulierung der Finanzmärkte wird auf eine „Weltregierung“ verschoben, welche die Gemüter in wissenschaftlichen Instituten in Wallung bringen kann, die aber angesichts der vorhandenen Widersprüche kaum in der Lage sein dürfte, die Finanzmärkte wirksam zu regulieren und anschließend zu kontrollieren.

Die großen Mitgliedsländer der Europäischen Union haben am 22. Februar 2009 einen Gipfel zur Vorbereitung des G 20-Gipfels, der im April die Strategie einer neuen Finanzmarktpolitik erarbeiten soll, veranstaltet, um eine europäische Position festzulegen. Schon in diesem Verfahren offenbarten sich die Legitimationsprobleme der EU, weil die Absprache neben den Institutionen und ohne die kleineren Mitgliedstaaten stattfanden. Das Ergebnis ist angesichts der Probleme ausgesprochen dürftig. Die Regierungen schwören weiter den Treueid auf den

11 Am 22. Juli 1944 schlossen die Industrieländer unter Führung der USA die Vereinbarung von Bretton Woods. Deren Ziel war ein stabiles Währungssystem und die reibungslose und von Handelsbarrieren befreite Abwicklung des Welthandels. Die Mittel, um dieses Ziel zu erreichen, bestand in der Vereinbarung fester Wechselkurse und einer Goldbindung des US-Dollars. Gleichzeitig wurden IWF und Weltbank als Steuerungsinstrumente für den Kreditverkehr und Aufbauhilfe für ein zerstörtes Europa etabliert. Der IWF sollte die Stabilität der Wechselkurse sichern, während die Weltbank für die Aufbauhilfe verantwortlich war. In der weiteren Entwicklung, insbesondere nach der Aufgabe der Goldbindung und der festen Wechselkurse, übernahmen diese Institutionen im Rahmen des von der WTO eingerichteten Freihandelsregimes eher unrühmliche Funktionen. Die Regulierung der Finanzmärkte und die festen Kontrollen, die mit dem System von Bretton Woods verbunden waren, waren das Fundament einer stabilen Entwicklung nach dem Zweiten Weltkrieg.

12 Merkel, Rede der Bundeskanzlerin auf dem Kolloquium "Neue Welt, neuer Kapitalismus", http:// www.bundesregierung.de/nn_774/Content/DE/Rede/2009/01/2009-01-08-merkel-kolloquium-kapitalismus.html. 
Freihandel: „Freier Handel“, heißt es in der Abschlusserklärung, „und Offenheit für grenzüberschreitende Investitionen sind wichtige Voraussetzungen dafür, dass die wirtschaftliche Dynamik im globalen Maßstab wieder zunimmt. "13 Wieder bleibt es dabei, dass die internationalen Institutionen, insbesondere der IWF, die wesentlichen Aufgaben übernehmen sollen. Das ist an sich nicht verkehrt, wenn es mit einer Bereitschaft verbunden wäre, in Europa zu eine Regulation der Finanzmärkte zurückzukehren und entsprechende Vorschläge für die internationalen Organisationen zu machen. Die Vorschläge bleiben aber in dieser Hinsicht bescheiden.

Gegen Steueroasen und „unkooperative Jurisdiktionen“ soll entschlossen vorgegangen werden. Wie das aussieht angesichts des Rückschrittsverbots bei der „Liberalisierung“ der Kapitalmärkte im Primärrecht? Zunächst soll ein Verzeichnis dieser „unkooperativen Jurisdiktionen“ erstellt werden, dann über Sanktionen nachgedacht werden. Und um keine Missverständnisse aufkommen zu lassen, wird als Zielrichtung gleich der internationale Terrorismus genannt. Damit sind die Finanzjongleure wohl nicht gemeint: Das heißt aber, ein Verbot von Kapitalexport in Off-Shore-Finanzmärkte wie die Cayman oder die KanalInseln steht nicht auf der Agenda der großen EU-Staaten. Ansonsten beschränkt man sich auf Kontrolle, Kooperation und ein Frühwarnsystem, nur die Bilanzvorschriften sollen antizyklischer gestaltet werden. ${ }^{14}$ Insgesamt bleibt es bei der Linie, mehr Transparenz zu schaffen.

Gegen den kurzen Anflug von Erkenntnis, dass der Markt Probleme schafft und nicht löst, wird damit weiter auf die Rationalität des Marktes gesetzt. Transparenz im Kredit- und Wertpapiergeschäft lässt sich doch nur insofern als Krisenregulation begreifen, als man davon ausgeht, dass der aufgeklärte Investor auf riskante Investitionen verzichtet. Nur dies scheint eben nicht der Fall zu sein, wenn mit dem Risiko ein entsprechend hoher Gewinn versprochen wird. Die strukturellen Zwänge innerhalb der Finanzmärkte sind durch Transparenz nicht zu bekämpfen. Nicht die Irrationalität der Akteure, sondern die Irrationalität der Logik des finanzmarktgetriebenen, deregulierten Kapitalismus führte in die Krise; eine Logik, der sich die handelnden Akteure nicht entziehen können.

Im Ergebnis ist die Europäische Union in ihren rechtlichen Grundlagen genauso wenig darauf eingestellt, eine Strukturreform des Weltfinanzsystems anzuschieben und umzusetzen, wie es die politischen Eliten sind. Angesichts des Ausmaßes der ökonomischen Verwerfungen könnte damit aber das Projekt der Europäischen Integration insgesamt in eine schwere Krise geraten.

\section{Versagen der Europäischen Union in der Wirtschaftskrise}

Neben die Festlegung auf „liberalisierte“ Kapitalmärkte tritt in der Union die Festlegung auf eine marktradikale Wirtschaftsordnung. Diese reicht vom grundsätzlichen Verbot von Beihilfen ${ }^{15}$ über das sog. Maastricht Defizitkriterium ${ }^{16}$ bis

Bundeskanzleramt - Zusammenfassung des Vorsitzes des G 20-Vorbereitungsgipfels in Berlin, file:// D:/0-Andis\%20Dateien/Oaktuelle\%20aufs\%C3 \%A4tze/finanzm\%C3 \%A4rkte/2009-02-22-zusammenfassung-g20-vorbereitung.html.

14 Ebd.

15 Dazu unter Vielen: Altmeyer, Gemeinschaftsrechtswidrige nationale Beihilfen, 1999; Cremer, Ökologische Kriterien bei der Vergabe öffentlicher Aufträge und EG-Beihilferecht - Materiellrechtliche und verfahrensrechtliche Aspekte, ZUR 2003, 265; Fischer, Öffentliche Aufträge im Spannungsfeld zwischen Vergaberecht und europäischem Beihilfenrecht, Vergaberecht 2004, 1.

16 Das Maastricht-Kriterium besagt, dass die Neuverschuldung $3 \%$ vom Bruttoinlandsprodukt nicht übersteigen und die Staatsverschuldung insgesamt nicht mehr als $60 \%$ des Bruttoinlandsprodukts betragen darf. 
zur Festlegung auf wettbewerbsorientierte Infrastrukturnetze (Art. 154 EGV =

170 AEUV), die zu einer „Liberalisierung“, d.h. Privatisierung von der Stromwirtschaft bis zum Eisenbahnverkehr ${ }^{17}$ geführt haben. Diese Regeln, die sich die Mitgliedstaaten in den Verträgen selbst gesetzt haben, werden gegenwärtig systematisch in ihr Gegenteil verkehrt oder schlicht gebrochen.

Die theoretische Vorstellung ist, dass staatliche Eingriffe in den Markt den Wettbewerb verzerren, der jedoch Voraussetzung eines gemeinsamen Binnenmarktes sei. Der Binnenmarkt wird als konkurrenzorientierte Veranstaltung gleicher Wettbewerber gedacht. Nach dieser Logik unterminieren staatliche Eingriffe diese grundsätzliche Gleichheit und führen zu Wettbewerbsvorteilen für die einzelnen nationalen Industrien. Nach der Ideologie der Verträge widerspricht das einer „offenen Marktwirtschaft mit freiem Wettbewerb, wodurch ein effizienter Einsatz der Ressourcen gefördert wird,“ so die marktliberale Begründung in Art. 98 EGV (120 AUEV). Nach dieser Konzeption ist es folgerichtig, dass Beihilfen grundsätzlich verboten und nur ausnahmsweise explizit zugelassen sind oder von der Kommission genehmigt werden können. Dabei geht der EuGH davon aus, dass Ausnahmevorschriften wie Art. 87 Abs. 3 b EGV eng auszulegen sind. ${ }^{18}$ Auf diese enge Auslegung hat sich die Kommission in ihrer Entscheidungspraxis ${ }^{19}$ durchgehend gestützt.

Mit der Wirtschaftskrise hat sich das Verhältnis verkehrt: Aus der Ausnahme ist die Regel geworden - selbstverständlich ohne dass die Vertragstexte geändert worden sind. Die Mitgliedstaaten subventionieren die Banken schrankenlos, es folgte die Autoindustrie und absehbar weitere Industriezweige. Die Zustimmung der Kommission ist eine Formalität, entsprechende Entscheidungen werden - im Grundsatz politisch richtig - am Fließband ${ }^{20}$ produziert. Weil sie keinen offenen Bruch riskieren will, formuliert die Kommission als neuen Grundsatz: „Angesichts des Ausmaßes der Finanzkrise und ihrer Auswirkungen auf das Wirtschaftsleben in den Mitgliedstaaten ist die Kommission der Ansicht, dass bestimmte Arten staatlicher Beihilfen für begrenzte Zeit gerechtfertigt sind, um die gegenwärtigen Schwierigkeiten zu überwinden. "21 Damit werden die 2004 strenger gefassten „Leitlinien der Gemeinschaft für staatliche Beihilfen zur Rettung und Umstrukturierung von Unternehmen in Schwierigkeiten" 22 gelockert und generelle Ausnahmeregelungen zusammengefasst und neu geschaffen. Die Wettbewerbsverzerrung wird, was sie ohnehin schon war: Ideologie. Auch wenn die Vorgehensweise im Prinzip richtig ist, bleibt es doch problematisch, die geltenden Regeln in ihr Gegenteil zu verkehren. Sie entpuppen sich in „ruhigen Zeiten“

Im Falle der Deutschen Bahn in Form der formellen Privatisierung, d.h. der Organisation eines Betriebes, der (bisher) in staatlichem Eigentum blieb, in privatrechtliche Organisationsformen. Die Ergebnisse sind allerdings ähnlich wie im Falle einer Eigentumsübertragung. Gewinn und nicht Versorgung mit einer günstigen Verkehrsinfrastruktur wird zum leitenden Organisationsprinzip.

18 Verbundene Rechtssachen T-132/96 und T-143/96, Freistaat Sachsen und Volkswagen AG/Kommission, Slg. 1999, II-3663, Rn. 167.

19 Entscheidung 98/490/EG der Kommission in der Sache C 47/96, Crédit Lyonnais (ABl. L 221 vom 8.8.1998, 28), Rn. 10.1, Entscheidung 2005/345/EG der Kommission in der Sache C 28/02, Bankgesellschaft Berlin (ABl. L 116 vom 4.5.2005, 1), Erwägungsgründe 153 ff., und Entscheidung 2008/263/EG der Kommission in der Sache C 50/06, BAWAG (ABl. L 83 vom 26.3.2008, 7), Rn. 166. Vgl. Entscheidung der Kommission in der Sache NN 70/07, Northern Rock (ABl. C 43 vom 16.2.2008, 1), Entscheidung der Kommission in der Sache NN 25/08, Risikoabschirmung WestLB (ABl. C 189 vom 26.7.2008, 3), Entscheidung der Kommission vom 4. Juni 2008 in der Sache C 9/08, SachsenLB (noch nicht veröffentlicht).

20 Vgl. die Statistiken der Kommission: http://ec.europa.eu/competition/elojade/state_aid/.

21 Mitteilung der Kommission - Vorübergehender Gemeinschaftsrahmen für staatliche Beihilfen zur Erleichterung des Zugangs zu Finanzierungsmitteln in der gegenwärtigen Finanz- und Wirtschaftskrise, 2009/C 16/01.

22 ABl. C 244 vom 1.10.2004. 
als Fesseln einer eher sozialstaatlichen Politik, die fallen gelassen werden, wenn der selbst angerichtete Schlamassel zu groß wird.

Als der Binnenmarkt mit einer einheitlichen Währung ausgestattet werden sollte, hatte vor allem die BRD Sorge um ihre stabile Währung, oder besser: die Relation niedrige Inflationsrate oder niedrige Arbeitslosigkeit wurde zugunsten der stabilen Währung entschieden; Arbeitslosigkeit wirkt so schön disziplinierend auf die Gewerkschaften. So wurde der Stabilitäts- und Wachstumspakt geschlossen, der den Mitgliedstaaten verbietet, sich höher als 3 \% des BIP neu zu verschulden. Sechs Mitgliedstaaten haben die Latte gegenwärtig gerissen: Frankreich, Spanien, Irland, Griechenland, Malta und Lettland; Deutschland wird spätestens im nächsten Jahr vertragsbrüchig. Im Unterschied zur letzten Flaute überschreiten die Staaten die Grenze aber nicht lediglich um Prozentpunkte hinter dem Komma. Irlands Neuverschuldung liegt bei ca. $10 \%$ des BIP. Da klingt es wie das berühmte Pfeifen im Walde, wenn die Bundeskanzlerin auf dem Brüsseler Sondergipfel Anfang März 2009 feststellt: "Der Stabilitäts- und Wachstumspakt ist das Fundament, auf dem die Wirtschaftspolitik der Europäischen Union ruht." Deshalb, folgert sie, „sollten wir die Dinge doch so abarbeiten, wie sie im Vertrag stehen und wie sie auch Teil des Maastrichter Stabilitäts- und Wachstumspakts sind. Wir können ja auch nicht einfach die Verträge ändern. “23 Genau dort liegt das Problem, die Verträge taugen nicht für eine Antikrisenpolitik.

Die Europäische Union ist auch in anderer Hinsicht wirtschaftspolitisch falsch aufgestellt für die Krise. Der Union fehlen die Steuerungsmittel, um angemessen auf die Krise zu reagieren, oder drastischer: Die Union ist auf eine Politik festgelegt, die Ursache der Krise war, und sie kann nur über Ausnahmevorschriften gegensteuern. Für eine antizyklische Konjunkturpolitik fehlen der Union schlicht die finanziellen Mittel und rechtlichen Kompetenzen. Im November 2008 verkündete die Kommission ein europäisches Konjunkturprogramm, um der „Rezession Einhalt zu gebieten“. Mit dem Programm sollte ein „massiver Kaufkraftschub für die Wirtschaft“ entwickelt werden. Dazu sollten umgehend Investitionen in Höhe von $200 \mathrm{Mrd}$. Euro getätigt werden. Schaut man aber genauer, dann trägt die Europäische Union davon schlappe $30 \mathrm{Mrd}$. Euro, und diese bestehen aus vorgezogenen oder umgeschichteten, nicht aus zusätzlichen Mitteln. „Vorgesehen ist eine sofortige Anschubfinanzierung in Höhe von $200 \mathrm{Mrd}$. EUR (1,5 \% des BIP der EU), die sich aus zusätzlichen Haushaltsmitteln der Mitgliedstaaten von $170 \mathrm{Mrd}$. EUR (ungefähr 1,2 \% des BIP der EU) sowie EU-Mitteln zur Unterstützung von Sofortmaßnahmen in Höhe von 30 Mrd. EUR (ungefähr 0,3\% des BIP) zusammensetzt“. ${ }^{24}$ Das Konjunkturprogramm ist in Wahrheit die Addition der Programme, die die Mitgliedstaaten sowieso initiiert hatten. Die Mitgliedstaaten agieren nach dem Motto „Rette sich wer kann“, legen nationale Rettungspakete und Konjunkturprogramme auf, die nur scheinbar von der Europäischen Union koordiniert werden. Um die Verhältnisse im Blick zu haben: Das deutsche „Rettungspaket“ für die Bänker und Anleger hat ein Volumen von 500 Mrd. Euro, das deutsche Konjunkturprogramm von 80 Mrd. Euro. Die Europäische Union hat keine Kompetenzen und keine Mittel, um eine eigenständige Konjunkturpolitik zu betreiben. Diese wäre aber nötig, wenn man die Sonntagsreden gegen den Protektionismus ernst nehmen wollte. So ist es nur verständlich, dass jeder Staat zusieht, dass seine Subventionen auch im eigenen Land bleiben. Da unterscheidet sich die Argumenta-

23 Pressekonferenz von Bundeskanzlerin Merkel zum EU-Sonderrat in Brüssel, http://www.bundesregierung.de/Content/DE/Mitschrift/Pressekonferenzen/2009/03/2009-03-01-pk-eu-sonderrat.html.

24 MITTEILUNG DER KOMMISSION AN DEN EUROPÄISCHEN RAT, Europäisches Konjunkturprogramm vom 26.11.2008. 
tion im Falle Opel nicht sonderlich von der im Falle Peugeot. ${ }^{25}$ Mit der USamerikanischen Begleitklausel zum Konjunkturpaket „buy american“ ${ }^{26}$ wird die Tendenz zu einem Paradigmenwechsel in der Weltökonomie sichtbar. Das heißt, die sich zu einer Systemkrise auswachsende Finanzkrise fördert in der Union Tendenzen der Re-Nationalisierung, die dem Umstand geschuldet sind, dass die europäischen Verträge keine wirksame Antikrisenpolitik der Union zulassen und die Mitgliedstaaten auf eine Politik festlegen, die - nun für jeden sichtbar - in die Katastrophe geführt haben. Auch hier schaffen die europäischen Verträge einen rechtlichen Rahmen, der die Handlungsfähigkeit der Union einschränkt. Dabei wehrt sich insbesondere Deutschland gegen eine Änderung der Rahmenbedingungen etwa durch eine Wirtschaftsregierung für die Eurozone, wie sie Sarkozy im Oktober 2008 forderte. $^{27}$

Die Durchsetzung freier Märkte in der Union bei gleichzeitiger Vereinheitlichung von Aspekten der Umwelt, des Verbraucherschutzes oder der Qualität bestimmter Produkte führten weiter dazu, dass eine Standortkonkurrenz zwischen den Mitgliedstaaten geschaffen wurde, die im Wesentlichen auf zwei Feldern ausgetragen wird, auf denen der Union relevante Kompetenzen fehlen: der Steuerpolitik und der Sozialpolitik. Für diese Politikfelder wird durch das Europarecht ein struktureller Druck erzeugt, der sich in einem "race to the bottom“ auf Seiten der sozialstaatlich organisierten Mitgliedstaaten verwirklicht. ${ }^{28}$ Diese Verpflichtungen führen in der aktuellen Systemkrise auf der Output-Seite zu erheblichen Problemen, die wie die genannten Elemente die Legitimität der Union zusehends untergraben. Die offensichtlich gewordene national-egoistische Vorgehensweise der Staaten manifestiert das wirtschaftspolitische Versagen der Union, das ihre Legitimationskrise verschärfen dürfte.

\section{Grundrechte und Umwelt wegwägen}

Die Legitimationskrise äußert sich mit Blick auf den Output und Input weiter in einer zunehmenden Skepsis gegenüber den Kompetenzerweiterungen oder -überschreitungen durch den EuGH, die hier nur kurz anhand seiner extensiven Auslegung der Grundfreiheiten erläutert werden sollen. Konnte die europäische Gurkennorm noch Gegenstand kabarettistischer Einlagen sein, bleibt dem Beobachter das Lachen im Halse stecken angesichts der Eingriffe des EuGH in das Arbeits- und Tarifrecht und im Ergebnis in den grundrechtlichen Schutz der Mitgliedstaaten. Im Namen der europäischen Wirtschaftsfreiheiten wie Dienstleistungs- und Kapitalverkehrsfreiheit hat der EuGH die auf langer Praxis beruhende Regulierung der industriellen Beziehungen, d.h. Streikfreiheit, Tarifrecht oder Mitentscheidungsrechte, eingeschränkt und einen Vorrang des europäischen Rechts gegenüber den Grundrechten der Mitgliedstaaten postuliert.

http://diepresse.com/home/wirtschaft/international/451134/index.do.

Siehe hierzu http://www.spiegel.de/politik/ausland/0,1518,604655,00.html.

Die Welt, 21.10.2008, http://www.welt.de/politik/article2604156/Sarkozy-fordert-Teilverstaatlichung-der-Industrie.html.

28 Huffschmid, Das „Europäische Sozialmodell“ - Nebelkerze oder politisches Konfliktfeld?, in: Viotto/ Fisahn (Hrsg.), Europa am Scheideweg - Kritik des EU- „Reformvertrags“, 2008, S. 68 (72). 
Bekannt geworden sind die Fälle Viking ${ }^{29}$ und Laval. $^{30}$ In beiden Fällen sah der EuGH einen Konflikt der Niederlassungsfreiheit und der in den Mitgliedstaaten grundrechtlich geschützten Vereinigungsfreiheit der Gewerkschaften, die das Recht umfasst, kollektive Maßnahmen wie Streiks oder Blockaden gegen Unternehmen einzusetzen. Die Niederlassungsfreiheit sei mit den Grundrechten im jeweils konkreten Fall abzuwägen. Im Falle Viking lässt der EuGH das Ergebnis offen und verweist die Abwägung an die nationalen Gerichte - allerdings ohne wirklichen Entscheidungsspielraum. Im Fall Laval gibt der EuGH selbst diese Zurückhaltung auf und prüft selbst die Verhältnismäßigkeit der kollektiven Maßnahmen, die er im Ergebnis für einen unverhältnismäßigen Eingriff in die Niederlassungsfreiheit hält.

Ein Zurückdrängen partizipativer Ansätze im Bereich der Ökonomie lässt sich auch an anderer Stelle beobachten: Die Kapitalverkehrsfreiheit wurde vom EuGH mit seiner Rechtsprechung zu den „Goldenen Aktien“ wie beim VWGesetz $^{31}$ ausgebaut zu einer Bastion gegen eine - selbst bescheidene - demokratische Kontrolle der Wirtschaft. ${ }^{32}$ Wenn ein gesetzlich bestimmtes besonderes Stimmrecht des Staates der Kapitalverkehrsfreiheit widerspricht, muss dies logisch auch für die Mitbestimmung von Arbeitnehmern oder sonstiger Öffentlichkeit gelten, die ganz ohne Aktien Stimmrechte haben.

Die Grundfreiheiten, das hat der EuGH angekündigt, machen auch vor der Garantie der menschlichen Würde nicht halt, sie wird zu einem prinzipiell abwägbaren Wert, ${ }^{33}$ der möglicherweise den Grundfreiheiten zu weichen hat. Das kann ganz praktisch werden im Bereich der Bioethik. Während das geltende Verständnis des Grundgesetzes der bioethischen Forschung und Anwendung gewonnener Erkenntnisse im Bereich der Humangenetik enge Grenzen setzt, weil der Schutz der menschlichen Würde absoluten Vorrang genießt, ist die Frage ungewiss, ob sich beispielsweise ein englischer Konzern in Deutschland nicht auf die Dienstleistungsfreiheit berufen könnte, um in Deutschland die engen Grenzen des Embryonenschutzgesetzes zu durchbrechen. Die schon ausgeurteilten Eingriffe des EuGH in die Koalitionsfreiheit veranlassten Fritz Scharpf zu fordern, solchen Urteilen den Gehorsam zu verweigern ${ }^{34}$ - wissend, dass damit die Legitimationskrise der Union offensichtlich wird.

Schließlich zeugen auch die Beschlüsse vom Dezember 2008 zur Klimapolitik von einer Legitimationskrise: nicht weil die ökologische Krise so nicht aufgehalten wird - das ist der andere, natürlich bedeutsamere Aspekt. Kommission und Parlament hatten versucht, die Ausschüttung von Emissionsberechtigungen so zu reduzieren, dass in der gegenwärtigen Emissionshandelsperiode Zertifikate

29 EuGH, v. 11.12.2007: Rs. C-438/05: Die finnische Viking Line wollte eines ihrer Schiffe umflaggen und in Estland registrieren lassen mit dem einzigen Zweck, das niedrige estnische Lohnniveau in Anspruch zu nehmen. Daraufhin hat die Internationale Transportarbeiter Föderation (auf Bitten der finnischen Gewerkschaft) der estnischen Gewerkschaft verboten, mit Viking Line zu verhandeln, was auch geschah. Das empfand Viking Line als Verstoß gegen die Niederlassungsfreiheit.

30 EuGH v. 18.12.2007, Rs. C - 341/05, Rn. 94: Das lettische Unternehmen Laval wurde mit Bauarbeiten in Schweden beauftragt. Entlohnt wurde nach dem lettischen Tarif, d.h. deutlich niedriger als in Schweden. Das veranlasste die schwedischen Gewerkschaften dazu, die Baustellen von Laval zu bestreiken, indem sie blockiert wurden. Auch hier meinte das Unternehmen, die Niederlassungsfreiheit sei unrechtmäßig eingeschränkt.

31 EuGH Rs. C-282/04; EuGH, Urteil vom 23. Oktober 2007 Rs. C-112/05.

32 Vgl. EuGH Rs. C-367/98; EuGH Rs. C-483/99; EuGH, Rs. C-503/99.

33 EuGHv. 11.12.2007, Rs. C-438/05, Rn. 46-Viking; EuGHv. 18.12.2007, Rs. C-341/05, Rn. 94 - Laval.

34 Scharpf, „Der einzige Weg ist, dem EuGH nicht zu folgen“, Interview in: Mitbestimmung $7+8 / 2008$, 19. 
einen Preis hätten bekommen können, ${ }^{35}$ der tatsächlich einen Anreiz dargestellt

hätte, $\mathrm{CO}_{2}$-Emissionen zu reduzieren. In der vergangenen Handelsperiode lag der Preis für eine Berechtigung bei einem Cent, weil zu viele Berechtigungen zu billig ausgegeben worden waren. Nachdem die Wirtschaftskrise nicht mehr zu übersehen war, haben die Regierungschefs der Union auf Druck der Industrie dafür gesorgt, dass ausreichend kostengünstige Zertifikate ausgeteilt werden. ${ }^{36}$ So soll bis 2013 die Menge an kostenfrei zugeteilten Zertifikaten einem Anteil von $80 \%$ entsprechen, danach die kostenfreie Zuteilung alljährlich abnehmen, so dass 2020 eine kostenfreie Zuteilung von $30 \%$ erreicht wird. Für Sektoren oder Teilsektoren, in denen ein erhebliches Risiko der Verlagerung von $\mathrm{CO}_{2^{-}}$ Emissionsquellen besteht, sollen die Zertifikate auch nach 2013 kostenfrei zugeteilt werden.

Das bedeutet faktisch einen erneuten Verzicht auf Preise im Emissionshandel, die Anreize zur Reduktion des Ausstoßes der Klimagase schaffen würden. Damit wird dem Versagen des marktgesteuerten Umweltschutzes die Krone aufgesetzt. Der Marktfetisch in der umweltrechtlichen Debatte dürfte mit den Brüsseler Beschlüssen den Todesstoß erhalten haben, aber die Union ist noch nicht in der Lage, sich auf andere Instrumente zu besinnen. ${ }^{37}$ Die ökologische Krise wird so in 2009 auch zu einer Krise der Legitimation des Marktansatzes der Union.

\section{Feblende demokratische Legitimität}

Probleme gibt es offensichtlich auch beim demokratischen Input. Das Demokratiedefizit ist in aller Munde; es hat zwei wesentliche institutionelle Komponenten, die durch den Vertrag von Lissabon nicht überwunden werden. Zunächst lässt sich feststellen, dass das Europäische Parlament weiterhin nicht der primäre Gesetzgeber in der Europäischen Union ist, sondern eher eine zweite Kammer, die den Rat kontrolliert. ${ }^{38}$ Das Europäische Parlament ist ein Parlament zweiter Klasse, das keine eigenen Gesetzesentwürfe auf den Weg bringen kann und auch Gesetzesentwürfe der Kommission nur mit deren Einverständnis abändern kann. ${ }^{39}$ Es ist ein Parlament zweiter Klasse, weil es kein Budgetrecht hat, wie es sich die Parlamente in den demokratischen Revolutionen erkämpft haben. Und es ist ein Parlament zweiter Klasse, weil es in vielen wichtigen Feldern der Politik nicht mitentscheiden kann, obwohl die Mitentscheidungsrechte durch den Lissaboner Vertrag ausgeweitet wurden. Die Handelspolitik etwa gerät durch den Vertrag in die ausschließliche Kompetenz der EU. Über Handelsabkommen, etwa zur Liberalisierung in den Bereichen des geistigen Eigentums, der Dienstleistungen oder zur Privatisierung der Wasserwirtschaft, wie sie im Rahmen der WTO immer wieder angestrebt wurden ${ }^{40}$ entscheidet der Rat ohne Parlament.

35 Fisahn, Vollzugsdefizite im künstlichen Markt, in: Altvater/Brunnengräber(Hrsg.), Ablasshandel gegen Klimawandel? Marktbasierte Instrumente in der globalen Klimapolitik und ihre Alternativen. Reader des Wissenschaftlichen Beirats von attac, S. 61.

36 RAT DER EUROPÄISCHEN UNION Brüssel, den 12. Dezember 2008 (OR. en) 17215/08; http:// www.campact.de/img/klima/EU-Paket/Gipfelbeschluesse.pdf v. 10.1.2009.

37 http://ec.europa.eu/environment/news/efe/eu_strategy/greenweek01_policymaking_de.htm.

38 So auch: Herzog/Gerken, Ein Beitrag zur EU-Verfassung, Welt am Sonntag v. 14.1.2007.

39 Zum Demokratiedefizit der Union und zur Stärkung des Parlaments siehe Fisahn/Viotto, Anforderungen an eine demokratische Union, ZRP 2007, 198 (200); siehe auch: Gusy, Demokratiedefizite postnationaler Gemeinschaften unter Berücksichtigung der Europäischen Union, in: Brunkhorst/Kettner (Hrsg.), Globalisierung und Demokratie, 2000, S. 131 f.; vgl. hierzu auch die Ausführungen bei MüllerGraf, Integration 3/2004, 186 (197); Streinz/Obler/Herrmann, Der Vertrag von Lissabon, 2008, S. 53.

40 Hilf/Oeter, WTO-Recht - Rechtsordnung des Welthandels, 2005, \$6 Rn. $19 \mathrm{ff}$.; vgl. insbesondere zur Wasserprivatisierung Höring, Wasser für Nahrung. Wasser für Profit, in: Brot für die Welt (Hrsg.), Hintergrundinformationen Nr. 15 (2005), 57. 
Gleiches gilt für die Liberalisierung des Kapitalverkehrs mit Drittstaaten, also zur Frage der De- oder Re-regulierung der Finanzmärkte. Der Rat bleibt das wichtigere Gesetzgebungsorgan, aber er ist nur höchst mittelbar legitimiert, besteht aus den Vertretern der Exekutive. Das Grundgesetz ist demgegenüber nach den geschichtlichen Erfahrungen - explizit und mit Recht skeptisch gegenüber Normsetzungen der Exekutive und hegt diese extrem ein.

Die oben erörterte wirtschaftspolitische Engführung der Verträge führt gleichzeitig zu einem demokratischen Problem: Wenn sich die Organisation einer demokratischen Gesellschaft durch die Möglichkeit des friedlichen Wechsels in der Regierung, durch das Spiel von Regierung und Opposition, von Mehrheit und Minderheit, die potenziell beständig die Rollen tauschen können, auszeichnet; ${ }^{41}$ wenn weiter Demokratie der friedlich ausgetragene Meinungskampf, die Auseinandersetzung um die Frage „Wie wollen wir leben?“ ist; wenn es darum geht, neue Mehrheiten für neue und andere politische Konzepte zu gewinnen und über Meinungs- und Pressefreiheit die prinzipielle Möglichkeit besteht, dass ein Politikwechsel möglich ist, dann können die konstitutionellen Grundlagen einer solchen Gesellschaft, und als solche sind die europäischen Verträge zu charakterisieren, nicht so angelegt sein, dass ein Politikwechsel ausgeschlossen ist. Während das Grundgesetz (bisher) wirtschaftspolitisch neutral ist, unterschiedliche wirtschaftspolitische Konzeptionen zulässt - eine angebotsorientierte, neoliberale Politik ebenso wie eine keynesianische, nachfrageorientierte Politik, eine den Marktgesetzen unterworfene Ökonomie ebenso wie Wirtschaftsdemokratie -, legen die europäischen Verträge mit den genannten und weiteren Elementen die Union auf eine angebotsorientierte, radikale Marktökonomie fest. Damit ist aber in einem wichtigen, vielleicht dem grundlegenden Feld der Politik ein Politikwechsel weitgehend ausgeschlossen und eine wesentliche Spielregel einer demokratischen Gesellschaftsordnung suspendiert.

Schließlich ist eine Legitimationskrise mit Blick auf die Verabschiedung des Lissaboner Vertrages als zukünftige Europäische Verfassung festzustellen. Die Krise äußerte sich zunächst in der missglückten Reform der Europäischen Union im Nizza-Vertrag 2001.42 Auch der folgende Verfassungsprozess scheiterte mit den ablehnenden Volksabstimmungen in Frankreich und den Niederlanden im Jahre 2005. ${ }^{43}$ Beim Ersatz für die europäische Verfassung, beim Lissaboner Vertrag, achtete die politische Klasse aller Mitgliedstaaten kollektiv darauf, dass er weder öffentlich diskutiert noch gar durch Volksabstimmung legitimiert werden muss. Allein Irland konnte nicht umhin, den Vertrag vom Volke abstimmen zu lassen - mit dem bekannten Ergebnis: das Vertragswerk wurde abgelehnt. Nun haben die Regierungschefs in Brüssel vereinbart, dass in Irland eine erneute Abstimmung über den gleichen Vertrag stattfinden soll. ${ }^{44}$ Das Volk darf solange abstimmen, bis das Ergebnis passt - die demokratische Substanz fehlt der Union. In Deutschland reagiert man darauf mit Denkverboten; an die Stelle des Nationalchauvinismus im Kaiserreich ist ein Europachauvinismus getreten. Kannte

41 Fisabn/Viotto, Anforderungen an eine demokratische Union, ZRP 2007, 198 (199).

42 Göler/Jopp, Der Konvent und die europäische Verfassung, in: Jahrbuch der europäischen Integration, 2002/2003, $35 \mathrm{f}$.

43 Vgl. hierzu Bos, Die Debatte über die Europäische Verfassung in den Niederlanden, in: Viotto/Fisabn (Hrsg.) (Fn. 28), S. 137 ff.; Herzog/Gerken, Europa entmachtet uns und unsere Vertreter, Die Welt v. 17.2.2007, http://www.welt.de/dossiers/eumacht/article720463/Europa_entmachtet_uns_und_unere_Vertreter. html; Khalfa, Brauchen wir eine europäische Verfassung, in: Viotto/Fisabn (Hrsg.) (Fn. 28), S. 17 ff.; Für eine europaweite synchrone Volksabstimmung über den Vertrag von Lissabon: Sauer,,Volksabstimmung über den Lissabonner Vertrag“, BayVBl 2008, 581 (585); Wuermeling, Die Tragische: Zum weiteren Schicksal der EU-Verfassung, ZRP 2005, 149.

44 Vgl.http://www.sueddeutsche.de/politik/458/451173/text/; http://www.euractiv.com/de/zukunft-eu/ eu-gipfel-gibt-irlands-forderungen-lissabon-vertrag/article-178010. 
Wilhelm II vor dem Ersten Weltkrieg nur noch Deutsche und keine Parteien mehr, so akzeptiert die hegemoniale Politik nur noch Pro-Europäer und tabuisiert die Frage: Welches Europa wollen wir denn?

Die Krise der europäischen Integration setzt dabei keineswegs erst mit der Finanzmarktkrise ein - diese macht sie nur offensichtlich. Sie ist vielmehr Folge der überhasteten Osterweiterung, ${ }^{45}$ die eher geostrategischen Überlegungen entsprang als dem bis dahin vorherrschenden Integrationsgedanken. Die Osterweiterung hat nicht nur die Abstimmungsmechanismen ${ }^{46}$ verkompliziert. Mit der Erweiterung geriet gleichzeitig das „Europäische Sozialmodell“ unter Druck, weil das Gefälle zwischen den „alten“ und den „neuen“ Europäern nicht mehr durch Finanztransfers ausgeglichen werden konnte. Der damit eingeleitete Krisenprozess äußert sich gegenwärtig in einer massiven Legitimationskrise, welche die gezeigten Komponenten hat und nach einer Neukonzeptionierung Europas verlangt, welche die Input- und die Output-Dimension der Demokratie stärkt.

\section{Finanzkrise aktuell}

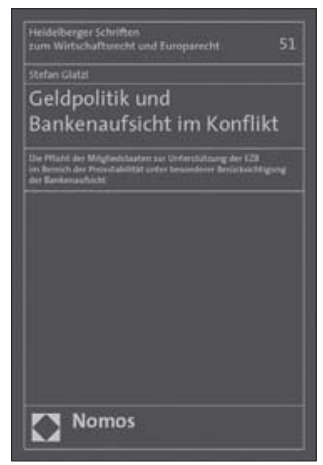

\section{Geldpolitik und Bankenaufsicht im Konflikt}

Die Pflicht der Mitgliedstaaten zur

Unterstützung der EZB im Bereich

der Preisstabilität unter besonderer

Berücksichtigung der Bankenaufsicht

Von RA u Stb Dr. Stefan Glatzl

2009, 350 S., brosch., 78,- $€$,

ISBN 978-3-8329-4175-8

(Heidelberger Schriften zum

Wirtschaftsrecht und Europarecht, Bd. 51)

Durch die weltweite Finanzkrise wird die Neuregulierung des Finanzwesens stark diskutiert. Das interdisziplinäre Werk beschreibt das rechtliche Fundament einer Neuordnung. Es stellt dabei die Wirkungszusammenhänge zwischen der Geldpolitik und der Bankenaufsicht in den Mittelpunkt und zeigt die Notwendigkeit und den Zwang zur Europäisierung der Bankenaufsicht auf.

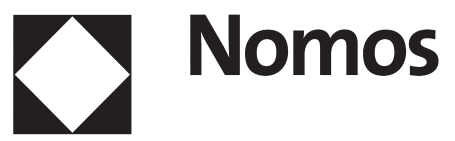

Bitte bestellen Sie im Buchhandel oder versandkostenfrei unter $\downarrow$ www.nomos-shop.de 